\title{
Análise das características de tráfego em redes ópticas comutadas por rajadas com processamento óptico de rótulos
}

\section{Traffic characteristics analysis in optical burst switching networks with optical label processing}

\author{
Fábio Durand; Felipe Rudge; Marcelo Abbade; Edson Moschim
}

\begin{abstract}
Resumo
Neste trabalhoérealizadaumaanálise deredesópticas comutadas por rajadasqueempregam processamento de rótulos compostos por códigos ópticos ortogonais (OOC) considerando as características de tráfego como comprimento/duração e taxa de chegada das rajadas. Os principais resultados mostram que a utilização do processamento de rótulos $\mathrm{OOC}$ atua na diminuição da probabilidade de perdas de rajadas, principalmente, para rajadas de pequena duração. Assim, pequenas rajadas que seriam bloqueadas nas redes com processamento eletrônico convencional são transmitidas quando se emprega o processamento de rótulos OOC. Dessa forma, ocorre um aumento de utilização da rede e uma diminuição na latência na transmissão das rajadas, alcançando uma granularidade próxima das redes de pacotes.

Palavras-chave: Códigos ópticos ortogonais. Redes ópticas comutadas por rajadas. OCDM (Optical Code-Division Multiplexing).
\end{abstract}

\begin{abstract}
An analysis is carried out with burst-switching optical networks which use label processing consisting of orthogonal optical codes (OOC), considering traffic characteristics such as length/duration and arrival rate of bursts. Main results show that the use of OOC label processing influences on the decrease of burst loss probability, especially for short-lived bursts. Therefore, short bursts that would be blocked in conventional electronic processing networks are transmitted when the OOC label processing is used. Thus, an increase in the network use occurs as well as a decrease in the burst transmission latency, reaching a granularity close to packets networks.

Key words: Optical orthogonal codes. Optical burst switching. OCDM (Optical Code-Division Multiplexing).
\end{abstract}

1 Docente do Curso de Engenharia Elétrica da Universidade Norte do Paraná - UNOPAR; fabio.durand@unopar.br

2 Docente da Faculdade de Engenharia Elétrica e Engenharia da Computação - FEEC - Universidade Estadual de CampinasUNICAMP

3 Docente da Faculdade de Engenharia Elétrica da Pontifícia Universidade Católica de Campinas, PUC-Campinas. 


\section{Introdução}

O constante crescimento da Internet está resultando em um aumento de demanda por altas taxas de transmissão e tecnologias de comutação que apresentem elevada velocidade de chaveamento (KANEDA, 2005) (MURTHY; GURUSAMY, 2002). Dessa forma, é necessário o desenvolvimento de redes de alta capacidade que sejam flexíveis para o compartilhamento de recursos entre diferentes tipos de serviços de forma eficiente. Uma solução tem sido a utilização de redes que integram o protocolo de Internet IP e a tecnologia da multiplexação por divisão de comprimentos de ondaWDM(Wavelength Division Multiplexing) (KANEDA, 2005). Porém, para tornar esta configuração eficiente, é preciso o emprego de tecnologias que permitam o roteamento e o encaminhamento de pacotes numa taxa de Tbps, para que haja compatibilidade com a velocidade de transmissão e roteamento da tecnologia WDM (BLUMENTHAL, 2000). Uma solução seria o emprego de redes ópticas de pacotes, visto que, nessas redes, o tráfego é transportado em pacotes ópticos juntamente com as informações de controle transportadas no cabeçalho óptico. O cabeçalho é extraído e processado eletronicamente em cada nó. Esse tipo de arquitetura apresenta a vantagem de possuir elevada utilização dos enlaces em função da multiplexação estatística e grande capacidade de adaptação ao tipo de tráfego oferecido à rede (YAO; MUKHERJEE, 2000). Porém, o custo e a tecnologia desse tipo de arquitetura ainda são proibitivos (MURTHY; GURUSAMY, 2002). Ademais, um dos principais desafios tem sido a busca pelo acesso aleatório a memória RAM (RandomAccess Memory) no domínio óptico (YAO; MUKHERJEE, 2000). Até o presente momento, o meio mais efetivamente utilizado como forma de armazenar as informações no domínio óptico por um tempo limitado são as fibras de linha de retardo FDL (Fiber Delay Line) que consistem de uma tecnologia limitada e inviável (YAO; MUKHERJEE, 2000). Assim, a tecnologia atual das redes ópticas não permite a comutação por pacotes e limita a atuação das redes somente por meio do estabelecimento de circuitos. O funcionamento das redes ópticas que empregam o paradigma da comutação por circuitos consiste em estabelecer caminhos ópticos entre os nós da rede. Esses caminhos ópticos são denominados lightpaths e são obtidos pela comutação de comprimentos de onda por meio de crossconects ópticos (LIU, 2002). Estas redes caracterizam-se por apresentar baixa granularidade e baixa eficiência de utilização, pois a sua unidade de transporte mínima são os comprimentos de onda. Este fato ocorre principalmente em função das características do tráfego atual transportado nas redes ópticas que consiste em sua maioria, ou $50 \%$ dos dados transmitidos, em pacotes menores que 522 bytes, sendo que $50 \%$ destes pacotes apresentam tamanho entre 40 a 44 bytes (BLUMENTHAL, 2000). Uma solução tem sido o emprego de redes comutadas por rajadas, também denominadas redes OBS (Optical Burst Switching) (QIAO, 1999). Nessas redes, o cabeçalho e as rajadas de dados são transportados em comprimentos de onda distintos. Dessa forma, o cabeçalho é processado eletronicamente nos crossconnects da rede para efetuar a reserva de recursos somente por um intervalo de tempo determinado. Assim, o caminho óptico estabelecido entre os nós de ingresso da rede é desconectado após o transporte da rajada. Essa separação entre o cabeçalho e os dados fornece às redes OBS uma grande flexibilidade e facilidade de gerenciamento (KANEDA, 2005). Esse tipo de rede também pode ser utilizada juntamente com o GMPLS (Generalized Multiprotocol Label Switching) para roteamento e sinalização para o estabelecimento dos caminhos ópticos (KANEDA, 2005) (BANERJEE, 2001), esta configuração também é conhecida como LOBS (Labeled-OBS) (MURTHY; GURUSAMY, 2002). No restante deste trabalh,o será empregado o termo rótulo para definir os pacotes de controle. Apesar das vantagens apresentadas pelas redes OBS, alguns estudos indicam que o atraso ocorrido em função do processamento eletrônico dos rótulos pode torna-se crítico em redes que trabalham com altas taxas de transmissão (KITAYAMA; MURATA, 
2003). Esse atraso se torna mais crítico para redes que cobrem pequenas distâncias e/ou para a transmissão de rajadas de pequeno comprimento. No intuito de aumentar a taxa de utilização dessas redes, estão sendo implementados recursos como grooming (FARAHMAND, 2003), roteamento por deflexão (LEE, 2003) e várias técnicas de montagem das rajadas (VOKKARANE; JUE, 2003), porém os resultados obtidos ainda não são satisfatórios (KITAYAMA; MURATA, 2003). Uma alternativa é o emprego do processamento óptico dos rótulos ópticos nas redes OBS como forma de aumentar a velocidade de processamento e a taxa de utilização destas redes. Este tipo de processamento foi inicialmente proposto em (KITAYAMA et al., 2002) e consistiu da utilização de pacotes de controle baseados em códigos ópticos coerentes da tecnologia de multiplexação por divisão de código OCDM (Optical Code-Division Multiplexing) (KITAYAMA; SOTOBAYASHI; WADA, 1999). Os códigos ópticos coerentes empregam a modulação de fase e intensidade e são mais complexos para implementar que os códigos ópticos não-coerentes, que utilizam somente a modulação em intensidade (MUTAFUNGWA; HALME, 2002). Dessa forma, em (DURAND; MOSCHIM, 2005a) foi proposta uma arquitetura de redes OBS que utilizava rótulos compostos por códigos ópticos não-coerentes denominados códigos ópticos ortogonais (OOC). Os códigos OOC são facilmente implementados com a atual tecnologia óptica e os resultados apresentados em (DURAND; MOSCHIM, 2005a) mostram que a utilização de rótulos ópticos OOC resulta no aumento da velocidade de processamento e aumento da taxa de utilização das redes OBS, de forma semelhante aos resultados apresentados em (KITAYAMA et al., 2002). Porém, ainda não foi descrito na literatura o desempenho de redes OBS com processamento de rótulos compostos de códigos ópticos, em função das características de tráfego como comprimento e taxa de chegada das rajadas.
Neste contexto, o objetivo deste trabalho é analisar o desempenho de redes ópticas OBS com rótulos de códigos ópticos OOC e verificar o desempenho desta rede em função das características de tráfego como comprimento/duração e taxa de chegada das rajadas. O restante deste artigo está organizado da seguinte forma. Na Seção II ilustra-se a arquitetura da rede analisada. Na Seção III é apresentada a metodologia de análise de desempenho desta rede. Na Seção IV apresentam-se os principais resultados e finalmente, na Seção V discutem-se as principais conclusões obtidas neste trabalho.

\section{Arquitetura e Funcionamento da Rede}

A arquitetura de rede OBS considerada neste trabalho emprega rótulos de códigos ópticos que são transmitidos no comprimento de onda destinado para controle (DURAND; MOSCHIM, 2005a). As informações transmitidas nos demais comprimentos de onda não empregam multiplexação OCDM. A seguir, ilustram-se algumas características de funcionamento das redes OBS com processamento convencional eletrônico dos pacotes de controle e apresentam-se as principais características da arquitetura empregada para o caso de utilização do processamento de códigos ópticos OOC.

\section{A. Redes Comutadas por Rajadas (OBS)}

Neste tipo de rede os dados são coletados nos nós de borda da rede e agrupados em rajadas de acordo com características como destino, classe de serviço $\mathrm{CoS}$ (Class of Service) e QoS, dependendo da arquitetura da rede (FARAHMAND, 2003). Esses nós OBS são responsáveis pelo estabelecimento dos mecanismos de conexão, a quais consistem em sinalização, roteamento e alocação de comprimentos de onda (BATTESTILLI, 2003). A Figura 1 ilustra um modelo simplificado de uma roteador de borda. 


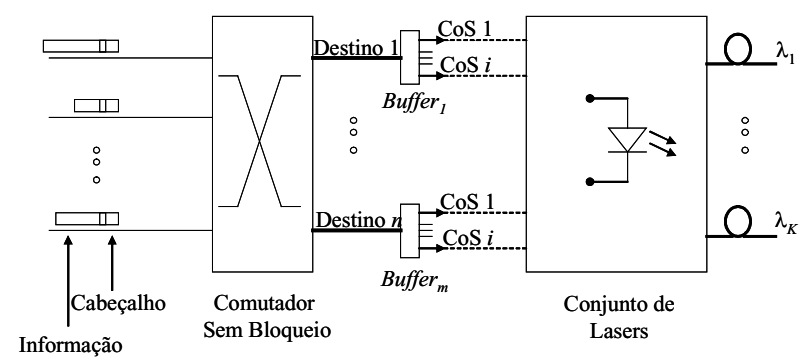

Figura 1. Modelo de roteador de borda utilizado.

A sinalização é utilizada para estabelecer e desconectar as ligthpaths reservadas, o roteamento é empregado para definir a trajetória que a rajada terá ao longo da rede e a alocação de comprimento de onda determina o comprimento de onda que será utilizado para a transmissão da rajada. A seguir serão analisados os tipos de sinalização empregados no de estabelecimento de conexão.

\section{B. Sinalização em Redes $O B S$}

A sinalização especifica o tipo de protocolo que será utilizado entre os nós da borda da rede para solicitarem acesso aos nós do núcleo da rede. Até o presente momento, os protocolos de sinalização podem ser classificados em duas categorias conhecidas como sinalização distribuída, comreserva em sentido único, e sinalização centralizada, com reserva fim-a-fim (KANEDA, 2005). Na sinalização distribuída com reserva em sentido único, existe a transmissão do rótulo pelo nó OBS de ingresso na rede, antes da transmissão da informação. $\mathrm{O}$ rótulo possui informações sobre a rajada e é processado eletronicamente no nó de ingresso e nos demais nós da rede. Este esquema facilita o controle eletrônico eficiente e permite que as informações transmitidas permaneçam inteiramente no domínio óptico. Dentre os protocolos que se enquadram nesta categoria pode-se citar o TAG (Tell-and-Go), JIT (Just-in-Time) e o JET (Just-Enough-Time) (YU et al., 2004) (QIAO, 1999). Na sinalização tipo TAG, ocorre a transmissão do rótulo pelo nó OBS de ingresso na rede antes da transmissão da informação para a reserva de recursos para a rajada com intervalo de tempo praticamente nulo (QIAO, 1999). O rótulo reserva recursos como comprimento de onda e FDL em cada nó da rota, pois enquanto o rótulo é processado eletronicamente, a rajada fica armazenada. Após completar a transmissão da rajada o nó de ingresso envia outro pacote de controle para a liberação dos recursos da rede. Na sinalização tipo JET existe a transmissão do rótulo pelo nó OBS de ingresso na rede antes da transmissão da informação para a reserva de recursos para a rajada (BATTESTILLI, 2003). O intervalo de tempo entre a transmissão do pacote de controle e da informação é denominada de TOffset. A Figura 2 ilustra este processo de reserva de recursos da rede em função do tempo. O rótulo possui informações acerca de a rajada como duração, destino, prioridade e é processado no nó de ingresso e nos demais nós da rede.

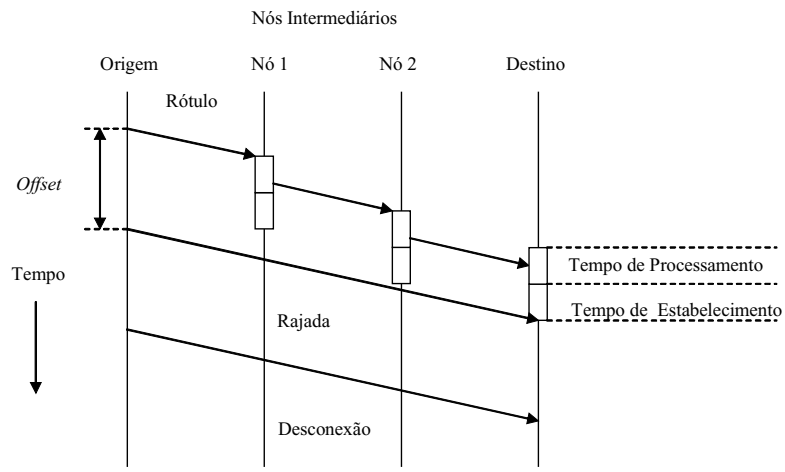

Figura 2. Processo de reserva de recursos de função do tempo empregando o protocolo de sinalização JET com processamento eletrônico dos rótulos

$\mathrm{Na}$ sinalização tipo JET ocorre a liberação dos recursos após o tempo previsto de utilização pela rajada, mas esse tipo de sinalização apresenta um efeito positivo na diminuição da probabilidade de perdas de rajadas (KITAYAMA; MURATA, 2003). Porém, ele apresenta a complexidade de necessitar do controle exato de todos os tempos envolvidos no processo, assim esta sinalização pode apresentar dificuldade de implementação em redes legadas (BALDINE, 2003). Uma alternativa é a sinalização tipo JIT, esta sinalização também se caracteriza pela transmissão do rótulo num intervalo de tempo antes da transmissão da rajada como na sinalização 
tipo JET. Entretanto após a transmissão da rajada é enviado um rótulo para efetuar a liberação dos recursos como ilustra a Figura 3.

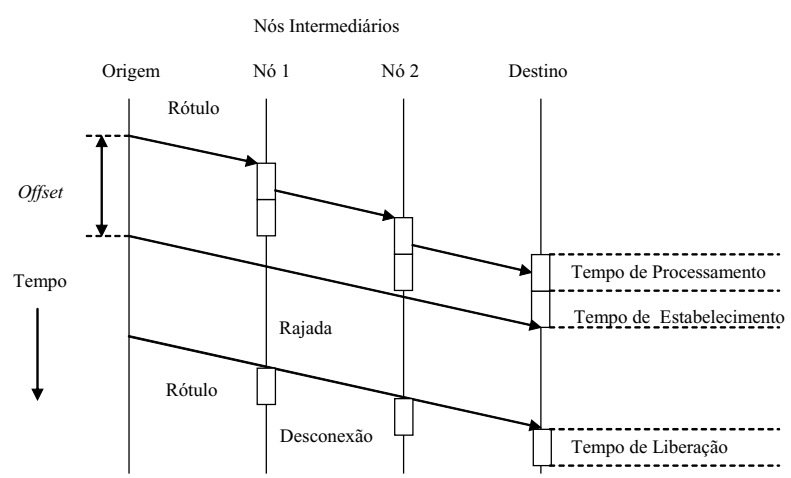

Figura 3. Processo de reserva de recursos de função do tempo empregando o protocolo de sinalização JIT com processamento eletrônico dos rótulos.

Apesar de aumentar a quantidade de rótulos narede, a sinalização tipo JIT requer menos complexidade com relação à predição dos tempos envolvidos no processo de sinalização (BALDINE, 2003).

\section{Redes Comutadas por Rajadas (OBS) com Processamento Óptico}

A rede OBS empregando processamento óptico de rótulos OOC será analisada neste trabalho em função das características de tráfego da rede. Ela emprega a arquitetura de processamento de códigos ilustrada na Figura 4 (DURAND; MOSCHIM, 2005a). Nesta arquitetura, ocorre a divisão de potência dos rótulos que chegam ao processador que fornece três cópias do rótulo, sendo uma utilizada para atribuição dos comprimentos de onda, outra empregada na determinação da rota estabelecida e a última cópia é utilizada na troca de rótulos. $\mathrm{Na}$ atribuição de comprimentos de onda e roteamento ocorre a correlação óptica dos rótulos. Neste processo será extraído o sinal que apresentar a maior correlação com o decodificador e a potência do código será convertida em sinal elétrico por meio de conversão eletro-óptica (EO), gerando sinais de controle que realizam a reserva dos recursos da rede, ou seja, reservam a posição de comutação e o enlace
WDM solicitados pelo roteamento da rede, até a chegada do rótulo com a mensagem de liberação dos recursos. A troca de rótulos é realizada no domínio óptico por meio de conversores ópticos de rótulos OOC que foram ilustrados em (DURAND; MOSCHIM, 2005b).

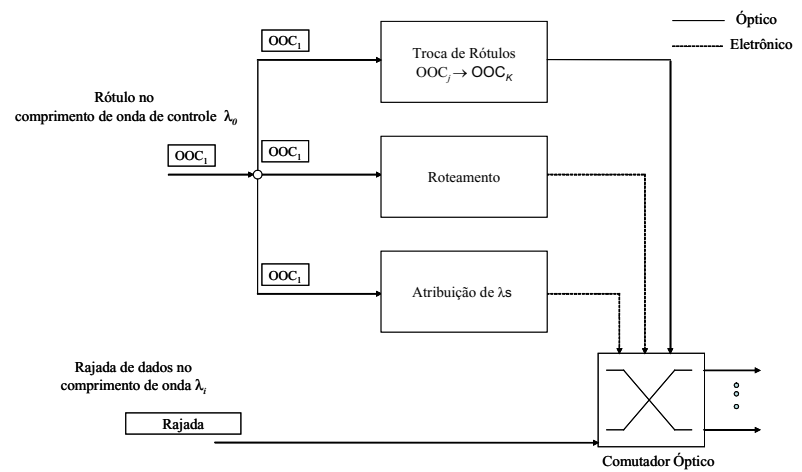

Figura 4. Arquitetura interna de leitura, troca de rótulos e comutação

O processamento óptico de rótulos possui como principal objetivo a diminuição do tempo de processamento óptico nos crossconnects do núcleo da rede. Será considerado o protocolo de sinalização tipo JIT porque a sua implementação é simples, se comparado com o protocolo JET, conforme foi ilustrado em (DURAND et al., 2006). Assim, como ilustra a Figura 5, o menor tempo de processamento diminuirá o tempo de TOffset entre a rajada e o rótulo para o caso do protocolo JIT.

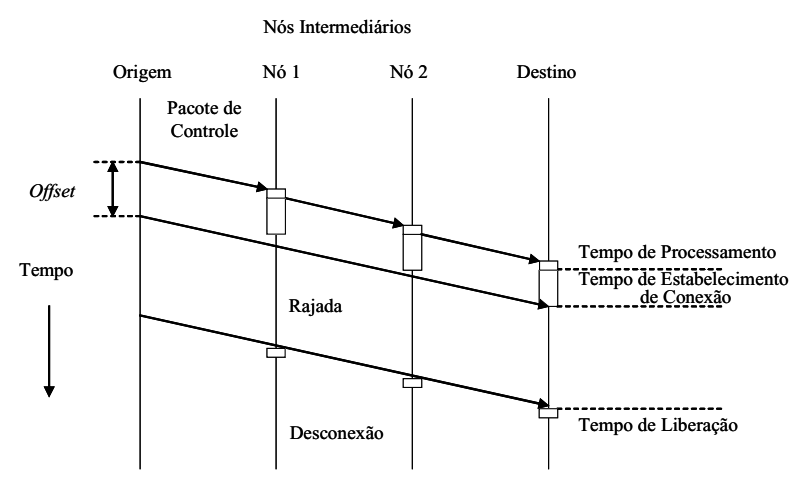

Figura 5. Processo de reserva de recursos de função do tempo empregando o protocolo de sinalização JIT com processamento óptico dos rótulos. 
Nos rótulos devem ser especificadas as informações sobre a rota e o comprimento de onda que serão reservados para as rajadas. Também deve existir a identificação do tipo de rótulo, ou seja, a definição se o rótulo é de conexão ou desconexão. Essa distinção pode ser realizada utilizando códigos OOC com famílias de códigos distintas. No emprego do protocolo JIT, os recursos ficam reservados até o rótulo com as informações de desconexão ser processado, para ocorrer a liberação dos recursos, como ilustra a Figura 5. As definições do rótulo de uma determinada rota e comprimento de onda são realizadas por meio do GMPLS, que estabelece tabelas para consulta de rótulos em cada nó da rede. Como o número de códigos ópticos é um recurso limitado em função do aumento da taxa de erro de bit que aumenta com número de códigos, é preciso otimizar as tabelas de rótulos para que o menor número possível de códigos seja utilizado. Por outro lado, os rótulos são definidos somente num enlace. Dessa forma, o conversor de códigos também auxilia a restrição da continuidade dos rótulos ao longo da definição da rota da rajada.

\section{Metodologia de Análise}

O modelamento matemático das redes OBS pode ser realizado por meio de métodos analíticos e numéricos (YU et al., 2004) (FARAHMAND, 2003). Neste trabalho, o modelamento da rede OBS será realizado de forma analítica, com o objetivo de ilustrar os efeitos da utilização do processamento óptico no dimensionamento e também no desempenho das redes OBS considerando as características do tráfego da rede. Inicialmente, serão determinadas as formulações para o cálculo do tempo de processamento óptico quando se empregam códigos OOC. Em (KITAYAMA; SOTOBAYASHI; WADA, 1999), foi ilustrado que o tempo necessário para a correlação óptica de códigos ópticos é dado por:

$$
T_{P}=\frac{1}{(L-1) / T_{C}}
$$

sendo $L$ o comprimento do código óptico e $T C$ o período de chip. Segundo (CHUNG, 1999) o comprimento $L$ é dado por:

$$
L=\lfloor C \cdot w \cdot(w-1)+1\rfloor
$$

sendo $C$ o número de códigos numa família de códigos com mesmo peso $(w)$. O peso de um código OOC é dado pelo número de chips "1" no código. Já $T C$ é dado por $1 / B L$, sendo $B$ a taxa de transmissão, resultando em:

$$
T_{C}=\frac{1}{B[C \cdot w \cdot(w-1)+1]}
$$

Como foi ilustrado anteriormente, uma grande parte do tráfego que é lançado nas redes é composto por pacotes com pequeno tamanho, assim a seguir será ilustrada a metodologia para analisar o efeito do comprimento das rajadas no desempenho da rede OBS.Considerando uma rede OBS saturada, a taxa média de chegada das rajadas num comutador óptico ( $\lambda$ OXC) é dada por (CALLEGATI, 1999):

$$
\lambda_{\text {OXC }}=F(W-k) \lambda
$$

sendo que $F$ é o número de fibras, $W$ é o número de comprimentos de onda por fibra, $k$ é o número de comprimentos de onda de controle e $\lambda$ é a taxa média de chegada das rajadas num determinado comprimento de onda. Neste caso, a taxa de chegada das rajadas será igual a taxa de chegada dos rótulos que é $1 / T P$, onde $T P$ é o tempo de processamento dos rótulos. Para obter a estabilidade da rede é preciso que: 


$$
T_{P}<\frac{(W-k) \lambda}{F}
$$

Portanto, a taxa média de chegada das rajadas deve ser limitada em:

$$
\lambda<\frac{(W-k) T_{P}}{F}
$$

Considerando o pior caso, no qual os comprimentos de onda estão sendo completamente utilizados, ou seja, $\rho=\lambda / \mu=1$, sendo $\rho$ a intensidade de tráfego, as rajadas devem ter uma duração mínima dada por:

$$
T_{\text {Rajada }}^{\min }=\frac{1}{\mu}=F(W-k) T_{P}
$$

Agora será analisado o esquema de reserva de comprimentos empregando a sinalização JIT. Neste esquema, deve-se considerar dois pontos importantes. O primeiro é a possibilidade de uma rajada se sobrepor ao tempo de TOffset de uma ou mais rajadas. $\mathrm{O}$ segundo diz respeito à disciplina empregada nos nós OBS que não prevêem prioridade para a primeira rajada que chega ao nó. $\mathrm{O}$ modelo empregado para analisar a porta de saída de um nó OBS será baseado nas seguintes considerações:

1. Os rótulos com informações de comutação, reservam recursos destinados a porta do comutador OBS que apresentam uma taxa de chegada, de acordo com um processo de Poisson com taxa média $\lambda$. Esta taxa de chegada equivale à taxa total sobre todas as portas de entrada do comutador. Esta consideração é comumente encontrada na literatura (YU et al., 2004) (FARAHMAND, 2003).
2. O comprimento das rajadas segue uma distribuição geral com CDF (Cumulative Distribution Function) B(l) e transformada de Laplace B*(s), sendo que $1 / \mu$ representa o comprimento médio.

3. O comprimento do Toffset das rajadas segue uma distribuição geral com CDF G(1) e transformada de Laplace $\mathrm{G}^{*}(\mathrm{~s})$, e Toffset representa o comprimento médio.

4. A taxa de chegada das rajadas também será considerada como um processo de Poisson com média $\lambda$. A maioria dos modelos analíticos emprega a consideração do tráfego na entrada da rede do tipo Poisson (YOO, 2000); Este tipo de tráfego é considerado adequado para redes com elevada taxa de requisição de transmissão de rajadas (YU et al., 2004).

A determinação do tempo de serviço efetivo de rajada para a sinalização JIT está indicada na Figura 6. Esta figura ilustra que para uma rajada, o comprimento de onda é reservado para a duração da rajada que é igual a soma de dois períodos de tempo. A duração do primeiro período é igual ao TOffset e é distribuído de acordo com a CDF $G(s)$ com média TOffset. Já a duração do segundo período é igual a duração da rajada e é distribuído de acordo com CDF $B(l)$ com média $1 / \mu$. Assim, a transformada de Laplace do tempo de serviço efetivo das rajadas é dada por $B(s) * G(s)$ com média (TOffset $+1 / \mu$ ) (DURAND et al., 2006).

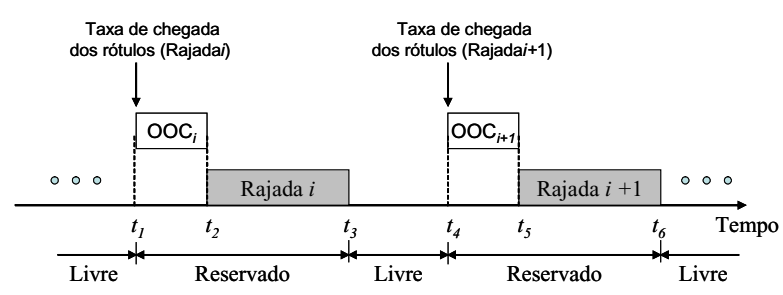

Figura 6. Diagrama de tempo do processo de reserva empregando JIT

Baseada nestas informações a porta de saída do nó OBS comporta-se como um sistema de perdas 
$M / M / K / K$, sendo $K$ o número de comprimentos de onda disponível na porta. A intensidade de tráfego é dada por:

$$
\rho=\lambda\left(\frac{1}{\mu}+T_{\text {Offset }}\right)
$$

O modelamento matemático analítico para o cálculo de perdas de rajadas apresenta boas características de precisão comparado com as simulações conforme ilustrada em (YU et al., 2004) e (YOO, 2000), principalmente para a sinalização tipo JIT. Para este tipo de sinalização, verificase que os resultados analíticos obtidos são muito aproximados dos resultados obtidos por simulação (DOLZER, 2001). Portanto, a porta de saída do nó OBS com $K$ comprimento de onda será modelada empregando um sistema $M / M / K / K$ e o limite superior da probabilidade de perdas de rajadas $P_{R}$ é determinada empregando a formulação de Erlang, com $r=\rho \cdot K$ :

$$
P_{R}=\frac{1 / K ! \cdot r^{K}}{\sum_{m=0}^{\mathrm{k}} 1 / m ! \cdot r^{m}}
$$

\section{Resultados}

Neste item são ilustrados resultados com intuito de analisar o impacto da utilização do processamento óptico em redes OBS, em função das características de tráfego. Inicialmente será analisada a economia de recursos da rede óptica OBS em função do emprego do processamento óptico dos rótulos. O recurso utilizado para análise são os comprimentos de onda empregados nos enlaces da rede. Neste estudo foi considerado o comprimento médio das rajadas $(1 / \mu)$ de $1 \mathrm{~ms}$, tempo de configuração do $\mathrm{OXC}\left(T_{O X C}\right)$ de 1 ms (SUN, 2005). O tempo de processamento óptico calculado por meio da Eq. (1) foi de 0,4 ns para uma taxa de transmissão de 2,5 Gbps, considerando uma família de códigos OOC com peso igual a 5 e com 10 códigos no comprimento de onda de controle (DURAND et al., 2006). Por outro lado, o tempo de processamento eletrônico dos rótulos considerado foi de $50 \mu$ s (SUN, 2005). Foram empregadas taxas médias de chegada ou solicitação de rajadas de $\lambda=$ $16 \mu$ e $\lambda=32 \mu$ para rotas com 5,10 e 15 enlaces ópticos. A análise realizada se baseia no número de comprimentos de ondas necessários para manter um determinado nível de probabilidade de perdas de rajadas. A probabilidade de perdas de rajadas é calculada por meio da Eq. (9) tanto para o caso do processamento eletrônico como para o caso do processamento óptico. No intuito de melhor ilustrar este conceito será empregada a métrica da economia relativa de comprimentos de onda, definida, neste trabalho, como:

$$
E_{W}(\%)=\frac{W_{\text {Electrônico }}-W_{\text {Óptico }}}{W_{\text {Electrônico }}} \times 100
$$

sendo $W_{\text {Eletronico }}$ e $W_{\text {Óptico }}$ o número de comprimentos de onda empregando o processamento eletrônico e processamento óptico, respectivamente. A Tabela I e Tabela II ilustram $E_{W}(\%)$ para as probabilidades de perdas de rajadas de $1 \times 10^{-2}$ e $1 \times 10^{-3}$ para taxas médias de chegadas de rajadas de $\lambda=16 \mu$ e $\lambda=$ $32 \mu$, respectivamente. 
Tabela 1. Economia de comprimentos de onda em função do emprego do processamento óptico dos rótulos para taxa média de chegada de rajadas de $\lambda=16 \mu$.

\begin{tabular}{cccc}
\hline $\begin{array}{c}\text { Probabilidade de perdas } \\
\text { de rajadas }\end{array}$ & 5 enlaces & $\begin{array}{c}\mathrm{E}_{W}(\%) \\
10 \text { enlaces }\end{array}$ & 15 enlaces \\
\hline $1 \times 10^{-2}$ & 8,3 & 15,4 & 22,8 \\
$1 \times 10^{-3}$ & 7,4 & 15,3 & 21,8 \\
\hline
\end{tabular}

Tabela 2. Economia de comprimentos de onda em função do emprego do processamento óptico dos rótulos para taxa média de chegada de rajadas de $\lambda=32 \mu$.

\begin{tabular}{cccc}
\hline $\begin{array}{c}\text { Probabilidade de perdas } \\
\text { de rajadas }\end{array}$ & 5 enlaces & $\mathrm{E}_{W}(\%)$ & 15 enlaces \\
\hline $1 \times 10^{-2}$ & 10,3 & 10 enlaces & 24,3 \\
$1 \times 10^{-3}$ & 9,4 & 16,2 & 23,7 \\
\hline
\end{tabular}

Por meio da Tabela I e Tabela II, verifica-se que a probabilidade de perdas de rajadas, tanto no emprego do processamento eletrônico como do processamento óptico, porém no emprego do processamento óptico ocorre uma economia do número de comprimentos de onda utilizados na rede OBS. Essa economia ocorre em função do menor tempo empregado pelo processamento óptico dos rótulos, pois ocorre a liberação da utilização dos recursos da rede para outras requisições de tráfego. Esse fato apresenta grande impacto na utilização da rede, principalmente para rajadas com tempo de duração semelhante ao tempo de comutação e configuração do OXC. Nos resultados, também se observa que a economia relativa de comprimentos de onda apresenta uma variação em função do número de enlaces percorridos na rota. Esse comportamento reflete o menor tempo empregado pelo processamento óptico, pois, conforme aumenta o número de enlaces percorridos pelas rajadas acarreta o impacto do tempo utilizado no processamento dos rótulos. Os valores obtidos na Tabela I e Tabela II são próximos, entretanto, o maior número de comprimentos de onda disponíveis para a maior intensidade de tráfego eleva a economia relativa de comprimentos de onda para os casos ilustrados na Tabela II. Até este ponto de análise dos resultados, foi ilustrada a vantagem do emprego do processamento óptico dos rótulos comparado com o processamento eletrônico no intuito da diminuição da probabilidade de perdas de rajadas e por conseqüência, uma economia nos recursos da rede, que neste caso são os comprimentos de onda. Portanto, os resultados sugerem que o processamento óptico pode ser empregado para aumentar a capacidade das redes OBS. Após verificar o efeito positivo do processamento óptico de rótulos na economia de recursos nas redes OBS, será analisado o seu efeito com relação às características de tráfego oferecido à rede. Neste contexto, um dos parâmetros que influenciam o projeto e o funcionamento de redes OBS é o comprimento das rajadas aceitas pela rede (YU et al., 2004), pois em redes OBS convencionais os pacotes de pequeno comprimento que chegam aos nós de ingresso são agregados com pacotes de mesmo destino para que a rajada tenha o comprimento adequado para ser transportada. Nos nós de borda são empregados algoritmos que definem as técnicas de agregação e preenchimento para a formação das rajadas. Na Figura 7, é ilustrada 
a duração mínima das rajadas permitidas em uma rede OBS em função da taxa de transmissão nos enlaces da rede tanto para o processamento eletrônico empregado em redes OBS convencionais como para o processamento óptico utilizado no caso de redes com rótulos compostos por códigos OOC. Neste trabalho serão considerados que os rótulos eletrônicos possuem 64 bytes de comprimento e é empregado um comprimento de onda para a sinalização.

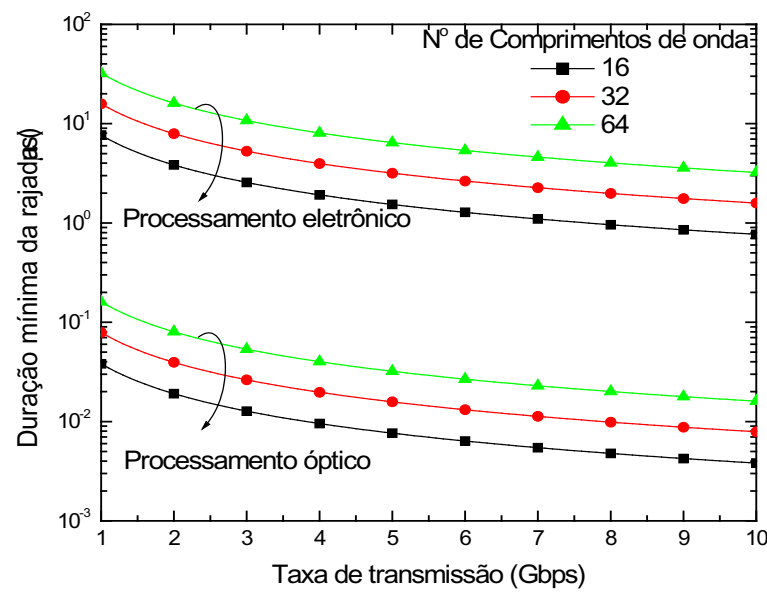

Figura 7. Duração mínima da rajada em função da taxa de transmissão considerado o processamento eletrônico e óptico dos rótulos para rótulos de 64 bytes e 1 comprimento de onda para controle.

Observe na Figura 7, que, ao empregar o processamento óptico de rótulos obtém-se rajadas aceitas pela rede com duração menor do que no caso da utilização do processamento eletrônico. Por exemplo, para o caso de redes com enlaces de 64 comprimentos de onda com uma taxa de transmissão de $10 \mathrm{Gbps}$, seriam aceitas rajadas com uma duração mínima de 3,2 $\mu$ s, no caso da utilização de processamento eletrônico das rajadas, por outro lado seriam aceitas rajadas com uma duração mínima de $0,01 \mu$ s no caso da utilização de óptico das rajadas. Em termos de comprimento das rajadas, seriam aceitas rajadas com comprimento de 4 kbytes e 12,5 bytes empregando o processamento eletrônico e processamento óptico, respectivamente. Dessa forma, observa-se que o comprimento das rajadas aceitas pela rede com processamento óptico é menor do que com processamento eletrônico. Portanto, empregando rajadas de menor comprimento na rede, haveria um aumento da granularidade e flexibilidade das redes OBS.

A seguir, será analisado o impacto do processamento óptico de rótulos na probabilidade de perdas de rajadas nos enlaces da rede OBS. Neste estudo, será considerado tempo de configuração do crossconnect (TOXC) de $1 \mathrm{~ms}$, tempo de processamento óptico de $0,4 \mathrm{~ns}$ para uma taxa de transmissão de 2,5 Gbps e tempo de processamento eletrônico foi de $50 \mu$ s (SUN, 2005). A Figura 8 ilustra a probabilidade de perdas de rajadas em função do comprimento médio das rajadas para uma taxa média de chegada ou solicitação de rajadas de $\lambda=1 / 62,5 \mu$ s para rotas com 5,10 e 15 enlaces, considerando o processamento eletrônico e o processamento óptico. Foram considerados 64 comprimentos de onda disponíveis.

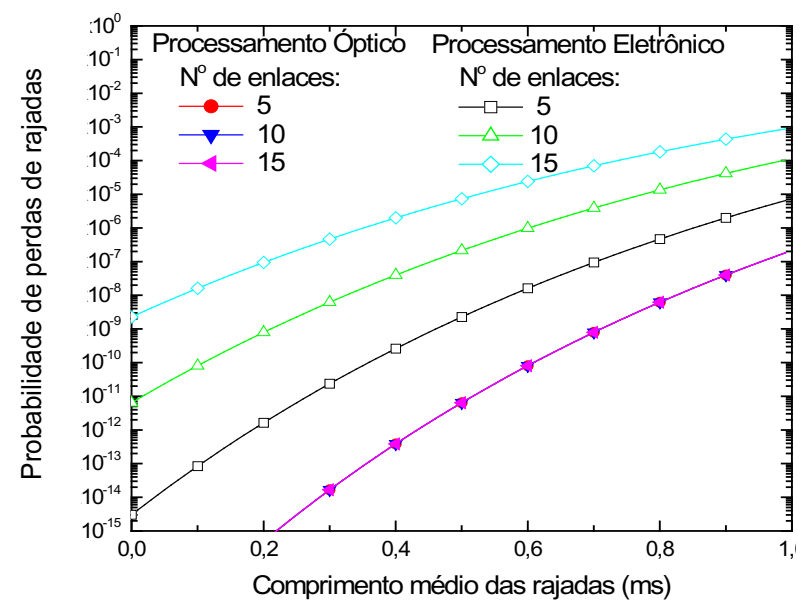

Figura 8. Probabilidade de perdas de rajadas em função do comprimento médio das rajadas para uma taxa média de chegada ou solicitação de rajadas de $\lambda=1 / 62,5 \mu$ s para rotas com 5, 10 e 15 enlaces, considerando o processamento eletrônico e o processamento óptico.

Por meio da Figura 8, observa-se que a probabilidade de perdas de rajadas aumenta em função do aumento do comprimento médio das rajadas. Porém, se verifica que a probabilidade de perdas de rajadas é menor quando se emprega o 
processamento óptico dos rótulos, principalmente para rajadas de pequeno comprimento/duração. Também se verifica que a probabilidade de perdas de rajadas não varia em função do número de enlaces na rota para o processamento óptico. Assim, observase que para rajadas de pequeno comprimento a probabilidade de perdas de rajadas empregando o processamento óptico é sensivelmente menor, quando comparada ao processamento convencional eletrônico. Com o intuito de verificar o efeito do processamento óptico em função do aumento da intensidade de tráfego será ilustrada na Figura 9 a probabilidade de perdas de rajadas em função do número do comprimento médio das rajadas para uma taxa média de chegada ou solicitação de rajadas de $\lambda=1 / 31,25 \mu$ s para rotas com 5,10 e 15 enlaces, considerando o processamento eletrônico e o processamento óptico. Os demais parâmetros empregados no estudo são os mesmos empregados para a obtenção da Figura 8, e apenas o número de comprimentos de onda que foi alterado para 128 .

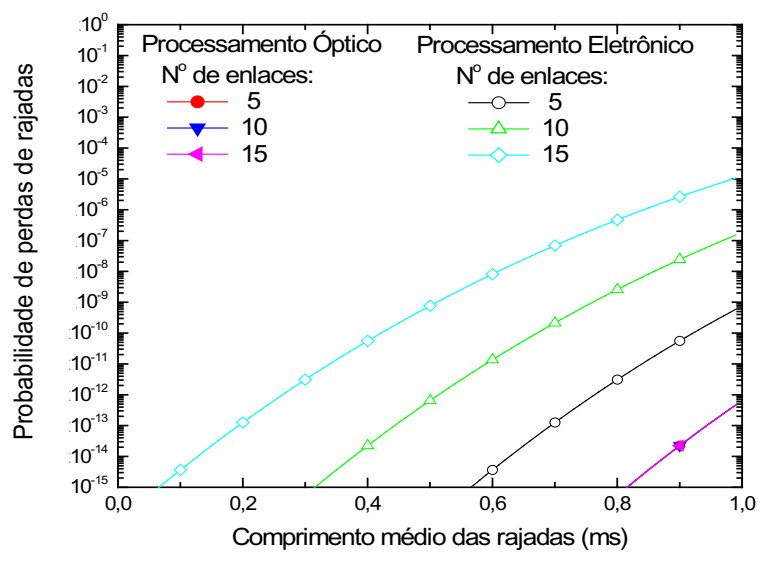

Figura 9. Probabilidade de perdas de rajadas em função do comprimento médio das rajadas para uma taxa média de chegada ou solicitação de rajadas de $\lambda=1 / 31,25 \mu \mathrm{s}$ para rotas com 5, 10 e 15 enlaces, considerando o processamento eletrônico e o processamento óptico.

Por meio da Figura 9 também se observa que a probabilidade de perdas de rajadas empregando o processamento óptico é sensivelmente menor quando comparado ao processamento convencional eletrônico para rajadas de pequeno comprimento. Para melhor ilustrar este resultado emprega-se o conceito de ganho de utilização ilustrado na Figura 10 em função do comprimento médio das rajadas. O ganho de utilização é definido como a razão entre a probabilidade de perdas de rajadas obtida pelo processamento eletrônico e a probabilidade de perdas de rajadas obtida pelo processamento óptico. Em termos práticos o ganho de utilização representa a liberação de recursos para serem utilizados pela rede OBS.

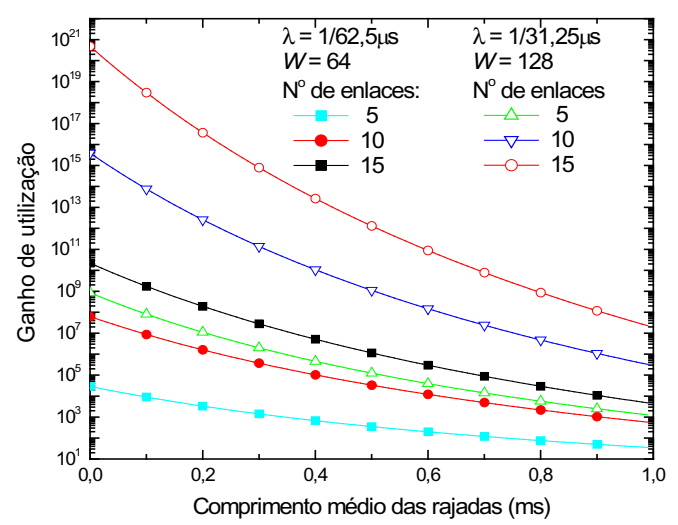

Figura 10. Ganho de utilização em função do número de comprimentos de onda para $\lambda$ igual a $1 / 62,5 \mu \mathrm{s}$ e $1 / 31,25 \mu$ s e rotas com 5,1015 enlaces.

A Figura 10 mostra que o ganho de utilização para rajadas de pequeno comprimento apresenta valores elevados, principalmente em redes com maior número de enlaces na rota. Dessa forma, verificase que as rajadas que seriam descartadas quando se emprega o processamento convencional eletrônico dos rótulos podem ser transmitidas na rede OBS empregando o processamento óptico. Os principais resultados obtidos mostram que a probabilidade de perdas de rajada empregando o processamento óptico é sensivelmente menor quando comparado ao processamento convencional eletrônico para rajadas de pequeno comprimento. Dessa forma, rajadas de pequeno comprimento que seriam bloqueadas em redes OBS com processamento convencional são transmitidas em redes OBS com processamento óptico. Uma análise global mostra que a rede 
OBS com processamento óptico de rótulos possui granularidade próxima a das redes comutadas por pacotes, porém sem as suas principais limitações tecnológicas.

\section{Conclusões}

Neste trabalho foi analisado o desempenho de uma rede OBS que emprega o processamento óptico dos rótulos considerando as características de tráfego como comprimento médio e taxa de chegada das rajadas. A rede OBS investigada emprega uma arquitetura de processamento óptico de rótulos que foi descrita considerando a utilização da sinalização de reserva de recursos. Emprega-se o protocolo JIT, por apresentar maior viabilidade de implementação, além de possibilitar a obtenção de resultados precisos quando se empregam modelos analíticos para a análise de desempenho da rede. Também foi investigado o tempo de processamento óptico obtido, empregando-se rótulos de códigos $\mathrm{OOC}$ e o comprimento mínimo das rajadas permitidas na rede. Os principais resultados obtidos mostram que a probabilidade de perdas de rajadas, empregandose o processamento óptico é sensivelmente menor quando comparado ao processamento convencional eletrônico para rajadas de pequeno comprimento. Dessa forma, rajadas de pequeno comprimento que seriam bloqueadas em redes OBS com processamento convencional são transmitidas em redes OBS com processamento óptico. Uma análise global mostra que a rede OBS com processamento óptico de rótulos possui granularidade próxima a das redes comutadas por pacotes, porém sem as suas principais limitações tecnológicas.

\section{Referências}

BALDINE, I.; CASSADA, M.; BRAGGT, A.; KARMOUS-EDWARDS, G.; STEVENSON, D. Justin-Time Optical Burst Switching Implementation in the ATDnet all-optical networking testbed." In: IEEE
GLOBECOM, 1-5 Dec. 2003, San Francisco, CA. Proceedings... New York: IEEE COMMUNICATIONS SOCIETY, 2003. p. 2777-2781.

BANERJEE A.; DRAKE, J.; LANG, J. P.; TURNER, B.; KOMPELLA, K.; REKHTER, Y. Generalized multiprotocol label switching: an overview of routing and management enhancements". IEEE Communications Magazine, New York, v. 39, n. 1, p. 144-150, Jan. 2001.

BATTESTILlI, T.; PERROS, H. An introduction to optical burst switching. IEEE Optical Communications, New York, n.8, p. 510-515, Aug. 2003.

BLUMENTHAL D. J.; OLSON, B. E.; ROSSI, G.; DIMMICK, T. E.; RAU, L.; ANOVI, M.; LAVROVA, O.; DOSHI, R.; JERPHAGNON, O.; BOWERS, J. E.; KAMAM, V.; COLDREN, L. A.; BARTON, J. All-optical label swapping networks and technologies. Journal of Lightwave Technology, New York, v. 18, n. 12, p. 20582075, Dec. 2000.

CALLEGATI, F.; CANKAYA, H.; XIONG, Y.; VANDENHOUTE, M. Design issues of optical IP Routers for internet backbone applications. IEEE Communications Magazine, New York, n. 12, p. 124-128, 1999.

DOLZER, K.; GAUGER, C; SPÄTH, J.; BODAMER, S. Evaluation of reservation mechanisms for optical burst switching. AEU-International Journal of Electronics and Communications, Wiesbaden, v. 55, n. 1, p. 1-6, 2001.

DURAND, F. R.; MOSCHIM, E. Uma arquitetura de rede OBS com labels de códigos opticos ortogonais. In: SIMPÓSIOBRASILEIRODETELECOMUNICAÇÕESSBrT, 22., 2005, Campinas. Anais... Campinas: Sociedade Brasileira de Telecomunicações, 2005a.

Analysis of hybrid WDM/OCDM networks with all-optical code converters. In: INTERNATIONAL MICROWAVE AND OPTOELETRONICS CONFERENCE, 2005, Brasília. Proceedings... Brasília: Cria Design, 2005 b.

DURAND, F.; ABBADE, M.; RUDGE, F.; MOSCHIM, E. Optical burst switching architecture with optical label processing. In: INTERNATIONAL TELECOMMUNICATIONS SYMPOSIUM, ITS, 6., 2006, Fortaleza. Proceedings... Fortaleza: SBrT, 2006.

FARAHMAND, F.; ZHANG, Q.; JUE, J. Dynamic traffic grooming in optical burst-switched networks. Journal of Lightwave Technology, New York, v. 23, n. 10, p. 31673177, 2003.

LEE, S.; KIM, H.; SONG, J.; GRIFFITH, D. A study on deflection routing in optical burst-switched networks. Photonic Network Communications, Wien, v. 6, n. 1, p. 51-59, 2003. 
LIU, K. H.; LIU, C. D.; PASTOR, J.; ROY, A.; WEI, A. Performance and testbed study of topology reconfiguration in IP over optical networks. IEEE Transactions on Communications, New York, v. 50, n. 10, p. 1662-1679, Oct. 2002.

KANEDA, S.; UYEMATSU, T.; NAGATSU, N.; SATO $K$. Network design and cost optimization for label switched multilayer photonic IP networks. IEEE Journal on Selected Areas in Communications, New York, v. 23, n. 8, p. 1612-1619, Aug. 2005.

KITAYAMA, K.; SOTOBAYASHI, H.; WADA, N. Optical code division multiplexing (OCDM) and its applications to photonic networks. IEICE Transactions Fundamentals, New York, v. E82-A, n. 12, p. 2616-2626, 1999.

KITAYAMA, K.; ARAKAWA, S.; OGUSHI, I.; MURATA, M. Ultrafast optical-code based path setup for optical burst switching. In: IEEE LASERS AND ELECTRO-OPTICS SOCIETY Summer Topics. Proceeding... Osaka: IEEE/LEOS, 2002. p.TuA2-21TuA2-22.

KITAYAMA, K.; MURATA, M. Versatile optical codebased MPLS for circuit, burst, and packet switchings. Journal of Lightwave Technology, New York, v. 21, n. 11, p. 2753-2764, 2003.

MURTHY, C. S.; GURUSAMY, M. WDM optical networks: concepts, design, and algorithms. New Jersey: UpperSaddle River, 2002.
MUTAFUNGWA, E.; HALME, S. Analysis of the blocking performance of hibrid OCDM-WDM transport networks. Microwave and Optical Technology Letters, New York, v. 34, n. 1, p. 61-68, 2002.

QIAO, C.; YOO, M. Optical burst switching (OBS)-a new paradigm for an optical internet. Journal of High Speed Networks, Amsterdam, v. 8, n. 1, p. 69-84, 1999.

SUN, Y.; HASHIGUCHI, T.; MINH, V.; WANG, X.; MORIKAWA, H.; AOYAMA, T.; Design and implementation of an optical burst-switched network testbed. IEEE Optical Communications, New York, n. 11, p. S48-S55, 2005.

VOKKARANE, W.; JUE, J. Prioritezed burst segmentatioan and composite burst-assembly techniques for QoS in optical burst-switched networks. IEEE Journal of Selected Areas in Communications, New York, v. 21, n. 7, p. 2062-2071, 2003.

YU, X.; LI, J.; CAO, X.; CHEN, Y.; QIAO, C. Traffic statistics and performance evaluation in optical burst switched networks. Journal of Lightwave Technology, New York, v. 22, n. 12, p. 2722-2738, 2004.

YAO, S.; MUKHERJEE, B. Advance in photonic packet switching: an overview. IEEE Communications Magazine, New York, n. 2, p. 84-93, 2000. 
